\title{
Returning students' perspectives on adjusting to medical graduate school in Korea: an interview study
}

\author{
So Youn Park, Oh Young Kwon and Tai Young Yoon
}

Department of Medical Education and Medical Humanities, Kyung Hee University School of Medicine, Seoul, Korea

\section{외국대학 출신 학생들의 국내 의학전문대학원 적응에 관한 질적 연구}

경희대학교 의학전문대학원 의학교육 및 의인문학교실

\section{박소연, 권오영, 윤태영}

Purpose: The recent trend of switching from medical graduate school to medical school in Korea raises questions about the adjustments that students must make in medical education. We examined the perceptions of medical graduate students with regard to their adaptation in medical education.

Methods: Sixteen semistructured, in-depth interviews were administered to medical graduate students who received their first degrees in foreign countries. The interviews addressed their perceptions of their experience in medical graduate school and on how well they adjusted to medical education.

Results: Students perceived their adaptation to medical graduate school in two dimensions: academic achievement and cultural adjustment. In academic achievement, a limited student-teacher relationship was recognized by students. Students tended to be passive in the classroom due to an uncomfortable atmosphere. They also reported witnessing culture shock in relation to the paucity of information on entrance into medical graduate school. Freshmen voiced many difficulties in adjusting to the unique culture in medical graduate school, in contrast to upper classmen. However, only $32 \%$ of students experienced helpful mentoring for their problems.

Conclusion: Students' perspectives should guide all decisions made about medical education in an altered educational system. Self-regulated learning and a good mentoring program can help prepare students for medical education and professional life.

Key Words: Adjustment, Medical students, Perception of medical education, Returnees

Received: September 24, 2014 • Revised: November 3, 2014 • Accepted: November 4, 2014 Corresponding Author: Oh Young Kwon (http://orcid.org/0000-0003-0817-2256)

Department of Medical Education and Medical Humanities, Kyung Hee University School of Medicine, 26 Kyungheedae-ro, Dongdaemun-gu, Seoul 130-701, Korea

Tel: +82.2.961.9102 Fax:+82.2.969.6958 email: koy0004@hotmail.com
Korean J Med Educ 2015 Mar; 27(1): 37-44. http://dx.doi.org/10.3946/kjme.2015.27.1.37 eISSN: 2005-7288

(C) The Korean Society of Medical Education. All rights reserved. This is an open-access article distributed under the terms of the Creative Commons Attribution Non-Commercial License (http:// creativecommons.org/licenses/by-nc/3.0/), which permits unrestricted non-commercial use, distribution, and reproduction in any medium, provided the original work is properly cited. 


\section{서론}

1996년 대통령자문 교육위원회가 처음 제안한 의학전문대 학원 제도의 도입 취지는 법학이나 의학 분야의 전문인들에 게 보다 높은 수준의 교양과 전문성을 제공함으로써 세계무 대에서 활발하게 활동할 수 있도록 하자는 것이었다[1]. 당시 기존의 의학교육은 다양한 인재를 양성하는 기능이 취약하다 고 비판 받았으며, 각 학교들은 대학의 다양화와 특성화 방법 에 대해 고민하였다[2]. 이에 따라 의학전문대학원 체제에서 는 이러한 요구에 부응하는 한 가지 방편으로 지원자들에게 다양한 입학기회를 제공하였으며 그 중 하나가 국외 소재 외 국대학에서 학사학위를 취득한 학생들을 선발하는 특별전형 이다. 한국의학교육 평가인증 규정에 따르면 학생 선발에 대 해 특성화된 대학원 편제에 합당한 다양한 경력을 소유한 학 생 선발 여부가 평가의 한 항목으로 포함되어 있으며[3], 이러 한 특별전형을 통해 현재 해마다 일정 비율의 외국대학 출신 학생들이 의학전문대학원에 입학하고 있다. 일례로 2011년 기준 주요 13 개 의학전문대학원 입학생의 외국대학 출신 합 격자 비율은 평균 $6.2 \%$ 였다[4].

의학교육 제도가 어떠한 형태이건 실제로 효과적인 의학교 육이 되기 위해서는 학습 활동과 학습 환경 그리고 학습자 사 이에 상호작용이 활발해야 하고 세 군 간에 균형이 잘 잡혀야 한다[5]. 특히 학습자는 교육의 주체이자 궁극적 수혜자이므 로 교육 과정에서 주도적인 역할을 하게 되는데, 동기 유발이 잘 된 학습자의 경우 교육 과정을 그 자체로 가치 있는 행동으 로 생각하고 보상과 상관없이 즐길 수 있게 된다[6]. 의학교육 을 받는 학생들의 학습 동기와 적응은 보상이나 유급 제도, 적 절한 도전과제와 같이 학교에 의해 제공되는 외적인 것들뿐 아니라 성별, 나이, 학사학위 소유 여부, 학생을 둘러싼 문화 적 환경 등의 요인들에 의해서도 영향을 받는다[7]. 그러나 이 제까지 우리나라 의학교육은 학업 성취의 효율성 측면에 치 중하여 교육 과정 개발이나 평가, 교수법 등에서 교육 제공자 에 중점을 두었으며 학습자 변인이나 그들의 시각에 대해 조 사하거나 평가하려는 노력은 미흡하였다[8]. 실제 의학교육 환경에 대한 학습자 관점을 알고 이해하는 것은 교육 제도 변 화를 결정하는 데 핵심적인 부분이며, 좀 더 의미 있고 효과적
인 의학교육이 되기 위해서는 학생들을 둘러싼 의료계 고유 의 문화 및 학교생활 적응에 대한 이해가 먼저 선행되어야 할 것이다[9]. 기존의 의학전문대학원 체제로부터 의과대학 체 제로의 전환이 가속화됨에 따라 이러한 연구의 필요성은 더 욱 대두되고 있다.

본 연구는 의과대학으로의 전환이 일개 대학에서만 일어나 는 현상이 아니라 전국의 상당수 의학전문대학에서 겪고 있 는 과정이라는 사실에 주목하여 의학전문대학원 체제 적응에 대한 학습자 관점을 알아보고자 한다. 의학전문대학원생 중 외국대학에서 학사학위를 취득하고 입학한 학생들은 개편된 교육 제도에 다양성을 제공한다고 여겨졌던 군이며 학위 취 득 기간 동안 한국의 교육에서 떨어져 있었기 때문에 한국의 의학 학습 환경이나 문화에 대해 좀 더 민감하게 반응할 수 있을 것이다. 따라서 이들의 시각을 통해 본 의학전문대학원 에서의 문제에 대한 분석은 의학 학습 기간 동안 학생들의 적 응 문제에 대해 교육적인 관심을 불러일으키고, 새롭게 변화 되는 교육 과정은 어떻게 운영할지 방향성을 잡는 과정이 될 것이며 동시에 그 동안 의학교육에서 부족했던 학습자에 대 한 기초자료를 제시할 수 있다는 점에서 그 의미를 찾을 수 있다.

좀 더 구체적으로, 본 연구는 외국대학에서 학사학위를 취 득하고 입학한 의학전문대학원생들을 대상으로 심층면접을 통해, 첫째, 학생들의 의학전문대학원 진학 동기를 파악한 후, 입학 당시와 비교하여 학업 성취와 적응에 대해 학생들이 어 떻게 인식하고 있는가를 분석하고자 한다. 둘째, 의학전문대 학원의 문화가 학교생활 적응과 학업 성취에 어떠한 영향을 미치는지 파악하고, 마지막으로 이에 대한 분석을 바탕으로 의학교육에서 학습자의 동기유발을 위해 어떠한 학습 환경을 제공해야 하는지에 대해 논해 보고자 한다.

\section{대상 및 방법}

이 연구는 2014년 7월에서 8월 말까지 약 2개월 동안 서울 소재의 한 의학전문대학대학원에서 외국대학 학사학위를 취 득하고 의학전문대학원에 입학한 1학년에서 4학년까지 16 명 을 대상으로 시행되었다. 의학교육 과정 적응에 대한 학생들 
의 인식을 알아보려는 연구의 목적을 감안하여 설문지 조사 와 같은 피상적인 형태의 자료보다는 깊이 있는 자료가 도움 이 되며, 학생들의 경험과 사고에 대한 복합적인 이해를 위해 서 연구자와 학생 사이에 상호작용이 필요하다고 판단했다. 따라서 질적 연구방법인 반구조화 심층면접(semistructured in-depth interview)을 시행하여 자료를 수집하고 분석하였 다. 면접을 통한 질적 연구는 각 면접자들이 그들의 상황에 대 해 어떻게 인식하고 있는지를 밝히는 데 도움이 되고 경험에 따른 결과 차이를 조사하는 데 유용하다[10]. 반구조화 심층 면접법은 최소한의 틀로 탐구되어야 할 주제들을 어느 정도 가정하고 시작하는 반면, 면담 대상자의 답변에 따라 개방형 질문을 사용하고 질문의 순서와 형태를 변화시킬 수 있는 개 방성 또한 갖추고 있다[11].

심층면접에서는 먼저 간략한 소개 후 기본적인 개인 배경 (학사학위 취득 국가)에 관한 질문으로 시작하였다. 이후 학 생들은 자유로운 분위기에서 다음의 개방형 질문들-의학전 문대학원 진학 배경(진학 목적과 이유, 대학원 선택 기준, 입 학 전 학교 지원 시스템), 대학원 생활(동아리 활동, 교우 관 계, 지도교수와의 관계), 학습 활동(학습 활동 시간, 한국어 강의 이해 정도, 학습 방해 요인), 의학전문대학원의 만족도 (만족 분야와 불만족 분야), 의학전문대학원에서의 성과, 졸 업 후 진로 계획-에 대해 대답하였다. 각 면접은 응답자들에 게 의학전문대학원에서의 적응을 위해 강화되어야 하는 부분, 학교나 교수에게 바라는 사항을 묻는 것으로 마무리 되었다. 면접 시간은 40 분에서 90 분까지 다양하였다.

\section{결과}

\section{1. 의학전문대학원 진학 동기}

연구 대상자로 면접에 참여한 학생들은 총 16 명으로 각각 1학년 5명, 2학년 1명, 3학년 4명, 그리고 4학년 6명이었다. 이들의 특성을 입학 배경별로 살펴보면 다음과 같다. 먼저 성별로는 남학생 7명, 여학생 9명이며, 학사학위를 받은 국 가는 캐나다 4 명, 미국 12 명이다. 이전 전공은 인문사회계열 이 3 명, 공학계열 1 명, 자연계열 12 명이다. 한국의 의학전문
대학원 진학 동기로는 더 좋은 취업 기회를 얻기 위해서거나 (9명), 다양한 학문적 경험을 위해 입학하였으며(3명), 기존 학업 수행 국가에서의 진학이 어려워서 택한 경우(2명), 그 리고 한국에 있는 가족과 지내고 싶어서 진학한 경우(2명)가 포함되어 있다. 학생들이 의학전문대학원에 대한 진학을 본 인 스스로 결정하였다는 사실은 기본적으로 의학전문대학원 진학 당시 학습에 대한 내적 동기를 가지고 있었음을 시사해 준다. 재학 중인 대학원을 선택한 배경에는 대학원의 순위나 평판도(7명), 교육 과정 및 교수진(1명)에 대한 고려 이외에 도 지인이나 친척의 추천(3명)과 입학조건(5명)이 영향을 미 쳤다. 본 연구에서는 면접 결과를 바탕으로 의학전문대학원 생들의 학교에 대한 적응을 크게 학업적 측면과 문화적 측면 으로 나누어 분석하였다.

\section{2. 의학전문대학원 적응 문제}

\section{1) 학업적 측면}

먼저 학업을 성취하는 데 걸림돌이 되는 구조적 원인으로 는 경직된 의대 학습 문화, 제한적인 교수-학생 관계, 부족한 학업지원 서비스 등이 지적되었다. 학습량이 방대한 의학의 특성상 빽빽하게 수업 스케줄이 정해져 있고 학생들은 강의 실에서 주로 수동적으로 수업에 임하기가 쉽다. 의학전문대 학원생들은 이러한 환경 속에서 위축감을 느끼거나 본인의 학습 능력에 대해 한계가 있다고 쉽게 규정하기도 하였다 (case 2, case 6).

한국사회 분위기인 것 같은데, 외국은 수업도중 잘 이해가 안 되는 부분은 “교수님 다시 한 번 설명해 주세요."라고 바로 그 자리에서 말하거든요. 그런데 여기에선 분위기가 경직되어 있어서 질문하는 것이 수업에 방해되는 것 같아 질문하기가 어려워요. 학업량도 많아서 의문을 가질 틈도 없이 교수님이 말씀하시면 '그런가보다.' 하고 넘어가요(case 6).

또 다른 요인으로는 제한적인 교수-학생 간 관계가 지적되 었다. 의과대학에서의 교수-학생 관계는 타 학과와는 달리 졸 업 후에도 인턴, 레지던트 과정을 통해 계속 유지될 수 있기 때문에 학생들은 교수들의 영향력에 대해 좀 더 심각하게 인 식하고 있고 사제 간의 관계를 좀 더 어렵게 느낀다. 외국에서 
학위를 받은 경험이 있는 의학전문대학원생들도 적극적으로 지도교수님들과 상담하는 경우는 전체 면담자의 $32 \%$ 에 지나 지 않았다. 상담을 경험한 학생들의 주된 상의 내용은 개인적 고민에 대한 내용과 향후 진로에 관한 내용이 주가 되었으며 그 외에 시험 및 성적에 대해 상의한다고 응답하였다. 그러나 상당수의 학생들이 학업 과정의 어려움에 대해 특별한 조언 이나 관심을 받지 못하고 스스로 해결하는 것으로 나타났다 (case 15, 16).

학교생활이 가끔 힘들 때도 있다는 것을 교수님들이 좀 알아 줬으면 $\cdots . . .$. 아무도 모르잖아요. 특히 1,2 학년 때는 하루 종 일 수업을 받아들이면서 ‘아, 이런 거구나.' 하고 있는 것이 너 무 힘들었어요(case 15).

1 학년 때 도서관에서 밤새면서 이게 뭐 하는 걸까 고민한 적 도 있고...... 버티면 지나간다는 느낌은 있는데 순간순간 힘 들었어요(case 16).

학과 강의를 어느 정도 이해하는가라는 질문에 대해서는 13 명의 학생이 $80 \%$ 이상 이해한다고 대답하였으며 나머지 3 명은 60\% 80\% 이해한다고 응답하였다. 수업의 이해를 어렵 게 하는 요인들로는 전공 특성상 내용이 어렵고 수업 진행속 도가 빠르기 때문이라는 답변이 가장 많았으며 그 외에 본인 의 기초학습이 부족하거나 예습, 복습을 하지 않아서라는 대 답이 뒤를 이었다. 학과 과제를 수행하는 데 소요되는 시간은 일주일에 평균 5 시간 이상에서 10 시간 미만이 소요된다는 응 답이 가장 많았다. 예습, 복습에 투자하는 시간은 개인별로 차 이가 컸는데 가장 많은 6 명의 학생들이 일주일에 평균 5 시간 이상에서 10 시간 미만이라고 응답하였으며, 그 뒤를 이어 4명 이 20시간 이상 투자한다고 대답하였다. 학업 만족도에 대한 질문에서 $19 \%$ 가 불만족한다고 대답하였으며 그 내용은 Table 1과 같다. 학업과 관련된 부분에서 특히 불만족하는 부 분은 장학금 혜택과 학업지원서비스, 그리고 학교 시설 및 교 육 여건 조성에 관한 부분이었다. 그 외에도 진로지도서비스 와 행정지원서비스에 대한 부분이 만족도가 낮은 것으로 확 인되었다.

\section{2) 문화적 측면}

학생들은 의학전문대학원의 문화가 상대적으로 위계질서 를 더 많이 강조하며 경직되어 있다고 인식하고 있었다. 또 민 감한 사안들(성적 발언이나 특정 계층 비하 발언)에 대해 특 별한 문제의식 없이 쉽게 언급하는 분위기도 문제로 지적되 었다.

문화 차이가 확실히 있는 것 같아요. 제 입장에선 이 정도 의 견을 제시해도 정당한 거라고 생각하는데 선배들이 밨을 때 는 '그건 아니지.'라고 하는 부분이 있어요(case 1).

이건 제가 조금 예민하게 받아들이는 것일 수도 있는데, 동아 리에서 선배들이 친해지려고 하는 것은 알겠는데 내가 이것 을 외국에서 들었다면 아니라고 했을 것 같은 성적인 발언들 을 만난 지 얼마 안 되어서 쉽게 하는 모습을 밨어요(case 1).

이러한 문화에 대해 입학 전에 어떠한 사전 정보도 받지 못 하고 있어 입학 후 문화 충격이 좀 더 심하다고 볼 수 있다. 특히 입학 직후인 1학년 때 문화충격이 가장 심한 것으로 나 타났지만 상급 학년으로 올라가면서 점차 적응해 가는 것으 로 인식하고 있었다. 흥미롭게도 학년이 올라갈수록 현재 문 화가 특별히 바뀔 것을 기대하기 보다는 본인이 적응해야 하 는 것으로 받아들이는 모습을 보였다.

이런 문화에 대해 알고만 들어왔어도...... 이 정도이다라고 솔직하게만 말해 줬어도 괜찮을텐데 예상을 못한 것들이 갑 자기 다가오니까 $\cdots \cdot \cdot(1$ 학년).

학교생활에 있어서 위계질서가 다른 집단보다 강하다 보니까 그것에 대해 적응 못하는 학생들이 있을 수 있어요. 이건 여 기 생리니까 변화시키기는 어려울 것 같고, 겪는 과정에서 잘 견딜 수 있도록 도와주어야 할 것 같아요(2학년).

학업적인 것만 해결이 되면 다른 부분들은 크게 문제가 되는 것 같지는 않아요. 솔직히 우리 학년에서 문화에 적응을 못하 는 것은 거의 못 봤어요. 3 학년 정도 되면 병원 등의 환경에서 어떻게 행동해야 하는지 알거든요(3학년). 
Table 1. Student Satisfaction and Dissatisfaction in Medical Graduate School: Themes, Frequency, and Sample Comments from Interviews

\begin{tabular}{|c|c|c|}
\hline Theme & Frequency & Sample comment \\
\hline \multicolumn{3}{|l|}{ Felt satisfaction with their school life } \\
\hline $\begin{array}{l}\text { Obtained knowledge and skills needed } \\
\text { for residency }\end{array}$ & 11 & $\begin{array}{l}\text { "In terms of knowledge, so far it is good. It has been a great to learn } \\
\text { new things" (case 7) }\end{array}$ \\
\hline Quality of teaching & 7 & $\begin{array}{l}\text { "I noticed that the problem (of academic achievement) is not the teacher } \\
\text { but my effort" (case 12) }\end{array}$ \\
\hline Exposure to medical practice settings & 5 & "It was nice to learn new skills for clinical practice through PBL" (case 3) \\
\hline Gain social network, including friends & 5 & "For me, it's great to have a chance to make friends" (case 8) \\
\hline Administrative support & 5 & $\begin{array}{l}\text { "I found that it was convenient to have administrative tasks over here" } \\
\text { (case 4) }\end{array}$ \\
\hline \multicolumn{3}{|l|}{ Felt dissatisfied with their school life } \\
\hline Hierarchical & 15 & $\begin{array}{l}\text { "Well, I guess they still follow old traditions (over here) and students stress } \\
\text { more on the hierarchy" (case 2) }\end{array}$ \\
\hline $\begin{array}{l}\text { Scholarship to study at medical graduate } \\
\text { school }\end{array}$ & 12 & $\begin{array}{l}\text { "Another thing I noticed was how hard it is to receive a scholarship. I } \\
\text { think this decreases my drive" (case 12) }\end{array}$ \\
\hline Lacking in academic support system & 12 & $\begin{array}{l}\text { "Each course has lots of content } \cdots \text { (even though they existed) I never } \\
\text { heard of a VOD lecture being offered" (case } 15 \text { ). }\end{array}$ \\
\hline Lacking in dormitory on campus & 9 & $\begin{array}{l}\text { "Because of insufficient facilities, I had to find a boarding house near the } \\
\text { school" (case 13) }\end{array}$ \\
\hline
\end{tabular}

Data in this table pertain to all 16 interviews.

PBL: Problem-based learning, VOD: Video on demand.

의학전문대학원 생활을 하면서 면담 학생의 $43 \%$ 가 차별을 느낀 적이 있다고 응답하였으며, 주된 내용으로는 성별에 대 한 차별이 가장 많았다. 학생들이 의학전문대학원에서 얻은 가장 큰 성과로 꼽은 것은 다양한 학문적 경험과 다양한 사람 들과의 교류였으며, 다음으로 더 좋은 취업기회 확보를 언급 하였다. 의학전문대학원 과정 중 생활에 대한 전체적 불만족 비율은 학업 불만족도에 비해 상대적으로 낮은 $6 \%$ 로 확인되 었다. 앞서 언급한 문화적 측면 이외에 특히 불만족스러운 부 분으로 지적된 것은 기숙사 시설의 부족이었다(Table 1).

졸업 후 계획에 대해 아직 특별한 계획을 세우지 못한 4명 을 제외하고 7명이 한국에서 수련 과정을 지속하겠다고 대답 하였으며 나머지 5 명은 외국에서 취업을 하고 싶다고 응답하 였다. 이러한 경향은 한국 문화에 적응하는 데 어려움을 겪었 던 경우 더욱 강하였다.

외국에서 사는 것에 대한 필요성은 한국에서 힘든 것이 어느 정도 반영된 것일 수도 있고. 여기 학교생활이 그렇게 만만한 것은 아니거든요. 한국에서 계속 학교를 다닌 학생들과의 차 이가 느껴져요. 적응하는 것도 그렇고, 시스템을 받아들이는 것도(case 12).

한국 와서 느낀 것이 나이가 좀 있는 상황에서 입학한 친구들 에게는 불이익이랄까? 갈 수 있는 곳도 좀 제한되어 있고 그 런 것이 있는 것 같아요. 외국에서는 전혀 그런 것을 못 느끼 다가 한국에서 그런 것을 느꼈어요. 그래서 외국의사자격 시 험을 보고 나갈까 해요(case 16).

\section{고찰}

본 연구에서는 외국대학 학위를 소지한 의학전문대학원생 들과의 심층면접을 통해 의학교육 과정 및 학교생활 적응에 대해 학업적 측면과 문화적 측면을 중심으로 살펴보고 의과 대학 교육에 대한 시사점을 도출하고자 하였다. 의학전문대 학원생의 경험에서 주목해야 할 사실은 교육 과정에 대한 적 응이 학생 개인의 문제이기도 하지만 상당 부분 의학전문대 학원 내의 구조적 요인들, 즉 경직된 의대 학습 문화, 제한된 교수-학생 관계, 수직적이고 다소 강압적인 문화와도 관련이 
Fig. 1. Self-Regulated Learning in Medical Education

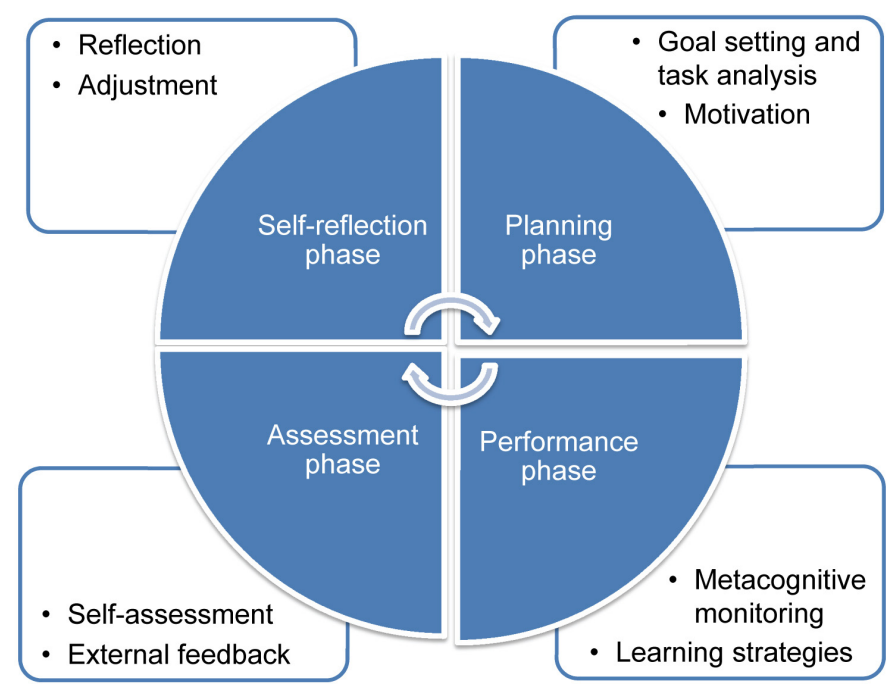

Adapted from Swanwick T. Understanding medical education: evidence, theory, and practice. Chichester, UK: Wiley-Blackwell; 2013 [5].

깊다는 사실이다. 학생들이 지적했던 부족한 부분들도 학습 환경을 둘러싼 구조적 요인들과 관련이 있었다. 여기서는 의 학교육에 대한 개선 방안으로 학습 문화 변화, 교수-제자 간 멘토링(mentoring) 프로그램의 활용을 제안하고자 한다.

의학전문대학생들은 기초의학, 인문사회의학, 임상의학에 관련된 지식뿐 아니라 전문가로서의 삶과 관련된 다양한 태 도, 술기, 임상 실습 등을 학습하게 된다. 이러한 교육 과정은 일반대학 학생들과 다른 적응을 요구하게 되며 그 과정에 요 구되는 학업강도와 학업량이 많은 편이다[12]. 하지만 심층면 접에 참여한 학생들 중 상당수가 학업 결과에 대해 본인의 책 임이라는 인식이 있으면서도 경직된 학습 문화로 인해 수동 적인 태도로 학업에 임하고 있었다. 성공적으로 의학교육 과 정에 적응하기 위해서는 교육 과정과 방법에 대한 이해를 바 탕으로 본인에게 가장 적합한 학습 방법을 선택할 수 있어야 하며, 한정된 자원과 시간의 효과적인 활용을 위해 스스로의 학습을 계획하고 평가하며 높은 동기를 유지해서 지속적으로 개선해야 한다. 이와 같은 특징은 메타인지, 학습 동기, 전략 적 행동을 포함하는 자기조절학습(self-regulated learning) 과 연관된다[13]. 의학교육에서 자기조절학습은 학습 계획, 학습, 피드백과 평가, 개선의 네 주기로 구성되는데[5], 먼저 학습 계획 단계에서 학생들은 주어진 학습 목표를 분석하고 자기효능감과 내적 동기를 바탕으로 학습 전략을 수립하게
된다(Fig. 1). 이는 자기조절(주의집중, 내용형상화 등의 방 법), 학습 전략 사용과 같은 학습수행 단계의 노력에 영향을 미치고, 이 과정은 다시 학습 결과에 대한 스스로의 평가를 거 쳐 학습자가 이를 어떻게 다음 학습에 반영하는 지까지 연결 되게 된다. Paris \& Paris [14]는 자기조절학습이 교육성과를 증대시킨다고 강조하였다. 이는 캐나다 의과대학생들을 대상 으로 한 연구에서 자기조절학습군이 대조군에 비해 척추천자 술기에 대한 시뮬레이션 연습 내용을 3개월 후에도 더 잘 유 지하고 있었으며 추후 이어지는 술기에서도 더 스스로에 대 해 자신감을 가지고 있었다고 보고한 결과와[15] 일맥상통한 다. 국내 의과대학생의 자기조절학습에 대한 연구에서도 메 타인지적 측면에서 더 적극적인 전략을 구사하고 행동적 측 면에서 본인의 시간이나 공부를 잘 조절하는 학생들이 진로 적응을 잘 하는 것으로 나타났다[12]. 그 동안의 연구들을 종 합하면 학생들은 교육을 통해 각 주기에 포함되는 전략적 방 법과 기술들은 성공적으로 습득할 수 있다고 보고되므로 $[5,14]$, 앞으로 의학교육에서는 학생들의 학업 성취와 의과대 학생활 적응을 위한 제도적 방안으로 자기조절학습능력 향상 과 관련된 다양한 상담과 중재 도입 등이 필요하겠다. 조금 더 구체적인 방안으로는 의과대학생들이 자기조절학습 과정 중 에서도 특히 목표 설정과 전략적 계획을 세우는 데 취약하다 는 이전 연구 결과를 감안하여[16], 학생들이 문제 해결에 있 
어 단순하고 초보적인 전략 수립에서 벗어나 좀 더 세분화된 목표를 세우고 그에 걸맞은 다양한 문제해결 방법을 사용할 수 있도록 하는 학습 전략 프로그램 개설이 있을 수 있겠다. 의학전문대학원 적응 과정 중 학생들의 입장이 크게 달랐던 분야는 멘토링 경험이었다. 정기적으로 멘토 교수님, 선배들 과 만남을 가지면서 학교생활에 대해 충분한 정보와 조언을 얻은 학생들은 동아리 생활이나 기타 학교생활에 수월하게 적응했다고 응답한 반면, 형식적인 1회성 만남을 가졌던 학생 들은 제대로 된 조언을 받지 못한 것을 아쉬워하였으며 학교 생활의 만족도도 낮았다. Radcliffe \& Lester [17]에 따르면 학생들은 입학 당시 등 가장 큰 변화가 있을 때 스트레스를 많이 받는다. 따라서 의대의 고유한 문화에 노출되는 충격에 대해 체계적으로 적응할 수 있도록 하는 프로그램이 필요한 데, 그 중 하나가 멘토링 프로그램이다. 멘토링은 경험이 풍부 한 멘토가 멘티의 전문성 개발뿐 아니라 인간관계 등 인격적 성장 지원, 진로 계획까지 지원하는 프로그램을 의미한다 [5,18]. 외국 의학교육에서 멘토링이 등장한 것은 1990년대 후반으로 그 역사가 짧으며 국내의 경우도 멘토링 프로그램 이 아직 효과적으로 운영되지 않고 있다[19]. 본 연구에서도 $68 \%$ 의 면접학생이 적응 문제에 대해 기존의 멘토 교수님이나 친구들에게 조언을 받지 못하고 있었다. 멘토링 프로그램을 운영한 국내 한 의과대학의 사례를 보면 프로그램에 대한 교 수들의 만족도보다 학생 만족도가 상대적으로 낮게 조사되었 다[18]. 학생들의 자기주도적 참여를 유도했음에도 불구하고 이러한 결과가 나온 원인 중 하나는 단기적 멘토링 프로그램 에 따른 한계점 때문인 것으로 해석된다. 교수들은 멘토링을 하면서 학생의 관심 사항과 고민에 대해 알 수 있는 시간이 되지만 멘티의 입장에서는 멘토링을 통해 단순한 파악을 넘 어서 실제로 도움이 될 만한 정보와 조언을 기대하게 된다. 따 라서 기존의 멘토링 프로그램이 학생들에게 실질적인 도움을 주기 위해서는 장기적인 프로그램으로 운영되면서 멘티의 욕 구와 의견이 충분히 반영되어야 한다[19]. 뿐만 아니라, 멘토 -멘티의 관계 특성상 프로그램 내에서 멘토의 역할이 그 결과 에 많은 영향을 미치는 것도 감안될 필요가 있다. 기존 연구에 서 학생들은 멘토링 과정을 형식적인 면담이라고 느끼는 경 우가 있었으며 교수의 시간, 경험 부족으로 크게 도움을 받지 못하는 것으로 조사된 바 있다[18]. 이를 극복하기 위해서는
멘토에 대한 전문적인 교육이 병행되어 전반적인 멘토링의 질 향상 및 관리가 되어야 할 것이다.

본 연구에서 의학전문대학원 학생들은 내적 학습 동기를 가지고 진학함에도 불구하고 경직된 학습 문화와 제한된 교 수-학생 관계, 고유의 위계질서 등에 대한 적응에 어려움을 겪고 있었다. 그러나 이에 대해 특별한 해결 방안을 찾고자 하 는 노력보다는 시간이 지나면서 익숙해지는 방향으로 순응하 고 있었다. 이에 앞으로의 의학교육 과정에서는 학생들의 학 업 자율성을 고양시켜 학습 그 자체를 즐길 수 있고 스스로를 학습의 주체라고 느낄 수 있는 환경을 제공하는 것이 필요할 것이다. 이 연구는 의학 과정 적응에 대한 연구로 단일 기관의 사례이고 특정 집단의 심층면접이기 때문에 변경될 제도 내 의 학생들의 입장과는 다를 수 있다는 한계점을 가진다. 그러 나 입학전형방법에 따른 차이는 의과대학 적응도에 영향을 미치지 않았다는 연구가 있으며[20] 이는 오히려 본 연구 결 과를 토대로 의과대학과 의학전문대학원 학생들의 적응과 의 과대학 교육목표 달성 여부를 분석하는 후속 연구를 요구한 다고 할 수 있겠다.

Acknowledgements: None.

Funding: None.

Conflicts of interest: None.

\section{REFERENCES}

1. Shin JS. A review on the courses of the introduction of post-baccalaureate basic medical education system in Korea. Korean J Med Educ 2006; 18: 121-132.

2. Park KH, Hong DH, Oh JH, Park YB, Shin DJ, Lee YD. The analysis of academic achievements of students at Gachon Medical School. Korean J Med Educ 2006; 18: 289-296.

3. Korean Institute of Medical Education and Evaluation. Medical college accreditation standards. Seoul, Korea: Korean Institute of Medical Education and Evaluation; 
2007.

4. Accreditation Standards [Internet]. Segyeilbo; 2011 [updated 2011 November 22; cited 2014 August 22]. Available from: http://www.segye.com/content/html/201 l/ 1 l/22/201 11 1 $22004948 . h t m l$.

5. Swanwick T. Understanding medical education: evidence, theory, and practice. Chichester, UK: Wiley-Blackwell; 2013.

6. Han JJ, Lee SN, Kwon I, Park H, Im HJ, Kim TE, An JH. The comparison of backgrounds and characteristics of students in medical college and graduate medical school: a case study of one medical school. Korean J Med Educ 2008; 20: 11-21.

7. Kusurkar R, Kruitwagen C, ten Cate O, Croiset G. Effects of age, gender and educational background on strength of motivation for medical school. Adv Health Sci Educ Theory Pract 2010; 15: 303-313.

8. Lee JU. Ideal reforming of curriculum in medical education. Chungnam Med J 2002; 29: 121-131.

9. Wolf SJ, Lockspeiser TM, Gong J, Guiton G. Students' perspectives on the fourth year of medical school: a mixed-methods analysis. Acad Med 2014; 89: 602-607.

10. Mason J. Qualitative researching. London, UK: Sage; 1996.

11. Dogra N, Giordano J, France N. Cultural diversity teaching and issues of uncertainty: the findings of a qualitative study. BMC Med Educ 2007; 7: 8.

12. Chun KH, Park EA, Song YM. The effects of medical students' self-regulated learning on career adaptability. J
Vocat Educ Res 2011; 30: 161-177.

13. Zimmerman BJ. A social cognitive view of self-regulated academic learning. J Educ Psychol 1989; 81: 329-339.

14. Paris SG, Paris AH. Classroom applications of research on self-regulated learning. Educ Psychol 2001; 36: 89101.

15. Brydges R, Nair P, Ma I, Shanks D, Hatala R. Directed self-regulated learning versus instructor-regulated learning in simulation training. Med Educ 2012; 46: 648-656

16. Artino AR Jr, Cleary TJ, Dong T, Hemmer PA, Durning SJ. Exploring clinical reasoning in novices: a selfregulated learning microanalytic assessment approach Med Educ 2014; 48: 280-291.

17. Radcliffe C, Lester H. Perceived stress during undergraduate medical training: a qualitative study. Med Educ 2003; 37: 32-38.

18. Kim J, Lee K, Hwang WM, Kang J. How to get students actively involved in course development: an experience in developing and implementing a mentoring program for medical students. Korean J Med Educ 2013; 25: $157-$ 165.

19. Hur Y, Kim S, Lee K. What kind of mentoring do we need? A review of mentoring program studies for medical students. Korean J Med Educ 2013; 25: 5-13.

20. Ryue SH, Lee HB. Korean medical students' cognitive, emotional, and social characteristics. Korean J Med Educ 2012; 24: 103-115. 